\title{
Further evidence on the similarity of memory processes in the process dissociation procedure and in source monitoring
}

\author{
MELANIE C. STEFFENS and AXEL BUCHNER \\ University of Trier, Trier, Germany \\ HEIKE MARTENSEN \\ University of Nijmegen, Nijmegen, The Netherlands \\ and \\ EDGAR ERDFELDER \\ University of Bonn, Bonn, Germany
}

\begin{abstract}
Buchner, Erdfelder, Steffens, and Martensen (1997) proposed that the memory processes involved in recognition judgments in the process dissociation procedure are the same as those involved in standard source monitoring tasks. Two extensions of that research are presented here. First, following a line of reasoning recently brought forward by Jacoby (1998), the instructions in the earlier study could have triggered different processes from those triggered by his instructions. However, with instructions conforming as closely as possible to those reported by Jacoby (1991), Experiment 1 replicated the earlier recognition judgment finding almost perfectly. Second, the memory processes underlying fame judgments in the process dissociation procedure could also resemble those involved in source monitoring tasks. Indeed, the results of Experiment 2 are compatible with the hypothesis that the same processes are involved in fame judgments given a source monitoring or a process dissociation procedure.
\end{abstract}

Jacoby (1991) proposed the process dissociation procedure for measuring the contributions of "controlled" and "automatic" processes to observable memory performance. The procedure has been very popular in memory research (e.g., Anooshian \& Seibert, 1996; Begg, Anas, \& Farinacci, 1992; Buchner, Steffens, Erdfelder, \& Rothkegel, 1997; Dehn \& Engelkamp, 1997; LeCompte, 1995; Neal, Hesketh, \& Andrews, 1995; Toth, Reingold, \& Jacoby, 1994; Yonelinas \& Jacoby, 1995). At the same time, numerous extensions and limitations have been discussed (e.g., Buchner, Erdfelder, \& Vaterrodt-Plünnecke, 1995; Cowan \& Stadler, 1996; Curran \& Hintzman, 1995; Dodson \& Johnson, 1996; Erdfelder \& Buchner, 1998; Jacoby, 1998; Jacoby, Begg, \& Toth, 1997; McBride \& Dosher, 1999; Mulligan \& Hirshman, 1997; Nelson, Bennett, \& Xu, 1997). The discussion that is of greatest interest for the present article focuses on the relation between (1) controlled and automatic memory processes as defined within the process dissociation procedure and

The research reported in this article was supported by grants from the Deutsche Forschungsgemeinschaft to A.B. (Bu 945/1-2) and to E.E. (Er 224/1-2). We thank Nelson Cowan, Peter Graf, John Reeder, and Jeffrey Toth for very valuable comments on an earlier version of this article, and Regina Grumann, Lars J. Jansen, Nikos Katris, Andreas Nack, Birgit Preiß, and Dirk Schmidt for their assistance with data collection. Correspondence should be addressed to $\mathrm{M}$. Steffens or A. Buchner, FBI-Psychologie, Universität Trier, D-54286 Trier, Germany (e-mail: steffens@uni-trier.de, buchner@cogpsy.uni-trier.de).
(2) source monitoring processes. Both Graf and Komatsu (1994) and Roediger and McDermott (1994) suspected that source memory could affect process dissociation procedure estimates of recollection and familiarity in recognition experiments. In the meantime, several articles have appeared in which it was empirically demonstrated that this may indeed be the case (Buchner, Erdfelder, Steffens, \& Martensen, 1997; Dodson \& Johnson, 1996; Komatsu, Graf, \& Uttl, 1995; Mulligan \& Hirshman, 1997). Mulligan and Hirshman introduced a model in which they distinguish between recollection that is diagnostic of list source and that which is nondiagnostic. Buchner, Erdfelder, et al. (1997) even argued that, more generally, the same memory processes are involved in performance in recognition tasks within the process dissociation procedure and in source monitoring tasks. They concluded that given the existing rich and detailed theoretical framework of source monitoring, the process dissociation procedure, when applied to recognition judgments, was redundant.

The experiments described in the present article extend the generality of Buchner, Erdfelder, et al.'s (1997) findings in two important ways. First, we were concerned that our earlier findings could have been partly due to our process dissociation instructions' stimulating source monitoring strategies. Second, up to now, all findings demonstrating a relation between source monitoring and process dissociation concerned recognition judgments. We extend our line of reasoning to cover the fame judgment task as well. 


\section{The Similarity Between the Process Dissociation Procedure and Source Monitoring}

When the process dissociation procedure is applied to a recognition memory paradigm, at least two classes of items are required in the acquisition phase. For instance, Jacoby (1991) had participants read words in Phase 1 (and solve anagrams) and hear words in Phase 2 . In a subsequent recognition test, participants responded to Phase 1 items, to Phase 2 items, and to distractors. In the inclusion condition, participants were instructed to call an item "old" if it was studied in Phase 1 or 2. Distractors had to be called "new." In the exclusion condition, participants were instructed to judge an item "old" only if it was presented during Phase 2 . In contrast, Phase 1 items had to be called "new" (i.e., they had to be "excluded"), along with the distractors.

According to Jacoby (1991), the inclusion condition represents a facilitation paradigm in which controlled recollection and automatic, familiarity-based processes operate "in concert" in such a way that an item is accepted as old on the basis of a controlled recollection or because of an automatic assessment of its familiarity. In contrast, the exclusion condition represents an interference paradigm in which the two process types are said to be put "in opposition." In this condition, participants try to reject the Phase 1 items whenever they can identify them as old. If they nevertheless accept such an item, then this is assumed to represent the operation of an automatic process contributing to item familiarity that could not be counteracted by a controlled recollection.

However, one could also reason that Phase 1 items are accepted in the exclusion condition because, although the item is recollected as an item that has been encountered in the experiment before, the necessary source information is not available (see Graf \& Komatsu, 1994). For instance, one may recollect that a certain word has been encountered in the experiment before, but simply misjudge it as a heard word from Phase 2 whereas it is actually a word that was read in Phase 1. This may be conceived as a classical example of source confusion.

In source monitoring investigations, the retrieval of source information is assessed by examining participants' source attributions (see Johnson, Hashtroudi, \& Lindsay, 1993, for a review). In the most simple case of a source monitoring procedure, participants classify items as having originated from Source A or B, or as being new. For instance, they may be asked, "Did you see the word in Phase 1 of the experiment (Source A), did you hear it in Phase 2 (Source B), or is it a new word?" If we assume that there is no difference in the processes contributing to recognition judgments given either process dissociation or source monitoring instructions, then it is easy to show how we may recover old and new responses for a process dissociation procedure recognition task from source monitoring data. This is illustrated in the left half of Table 1. The first row in Table 1 illustrates that whenever participants consider a word as a Phase 1 word, they will respond "Phase 1" in a source monitoring task, "old" in the inclusion condition of the process dissociation procedure, and "new" in the exclusion condition of the process dissociation procedure (because Phase 1 words must be "excluded"). If they classify a word as a Phase 2 word, they will respond "Phase 2" in a source monitoring task and "old" in both the inclusion and exclusion conditions of the process dissociation procedure. Finally, if participants think of a word as a distractor, they will respond "new" in a source monitoring task as well as in both test conditions of the process dissociation procedure. Thus, we may assume that participants in the source monitoring condition would have responded "old" instead of "Phase 1" had they been given inclusion test instructions, and they would have responded "new" instead of "Phase 1 " had they been given exclusion test instructions. Additionally, they would have responded "old" instead of "Phase 2" had they been given inclusion or exclusion test instructions.

In order to test whether this reasoning is valid, Buchner, Erdfelder, et al. (1997) took the response frequencies obtained in a source monitoring task and reconstructed the response frequencies as they would have resulted from a process dissociation experiment, using the mapping rules illustrated in Table 1 . These reconstructed frequencies were then used to estimate the parameters representing recollection and familiarity in the extended measurement model for the process dissociation procedure (see Buchner et al., 1995). The memory parameters as estimated from the reconstructed response frequencies were indeed equal to the memory parameters as estimated from genuine process dissociation response frequencies. What is more, even the raw frequencies for Phase 1 words and distractors obtained in the genuine process dissociation groups did not differ significantly from those obtained in the reconstructed process dissociation groups. Buchner, Erdfelder, et al. (1997) thus concluded that the process dissociation procedure memory parameters cannot be differentiated from those reconstructed from source monitoring data. The similarity of the raw frequencies implies that their findings did not depend on the type of measurement model used. However, their findings may have depended on the specific instructions they gave to participants in the process dissociation exclusion group.

\section{Effects of Instruction}

Recently, Jacoby (1998, p. 21) argued that in applications of the process dissociation procedure, "details of instructions serve as an important boundary condition for findings." He demonstrated that the details of the test instructions and procedure may induce either a directretrieval or a generate-recognize strategy, the latter of which Jacoby classified as a strategy for which the process dissociation procedure was not applicable. Jacoby (1999) also argued that subtle procedural differences may encourage the use of different strategies and thus lead to different results. A problem similar to that addressed by Jacoby (1998) may have been inherent in the Buchner, Erdfelder, et al. (1997) experiment. Participants in the exclusion condition were informed that only those words 
Table 1

Illustration of the Relation Between Response Categories in a Source Monitoring Task and the Response Categories in the Inclusion and Exclusion Conditions in the Process Dissociation Procedure for a Recognition Judgment Task and a Fame Judgment Task

\begin{tabular}{llc}
\hline \multirow{2}{*}{$\begin{array}{c}\text { Source } \\
\text { Monitoring }\end{array}$} & \multicolumn{2}{c}{ Process Dissociation Condition } \\
\cline { 2 - 3 } & \multicolumn{1}{c}{ Inclusion } & Exclusion \\
\cline { 2 - 3 } Phase 1 & Old & New \\
Phase 2 & Old & Old \\
New & New & New \\
& & Fame Judgment Task \\
Study phase & Famous & Nonfamous \\
Famous & Famous & Famous \\
Nonfamous & Nonfamous & Nonfamous \\
\hline
\end{tabular}

that had been heard before counted as old. Subsequently, this instruction was explained in detail: They were asked (1) to respond "new" to words they had earlier read or solved as anagrams; (2) to respond "new" to words they had not encountered in the experiment before; but (3) if they had heard a word in Phase 2, it should be classified as old.

The problem is that these instructions could be conceived of as being relatively close to typical source monitoring instructions in that they explicitly pointed to the fact that three types of items had to be distinguished. Moreover, the design of the instruction screen made the distinction between these three types of items maximally obvious. We chose these instructions in an effort to make the exclusion test instructions as easy to understand as possible, given that large proportions of participants had misunderstood the exclusion instructions in prior experiments (see Graf \& Komatsu, 1994). At that time, we were unaware that the details of instructions might alter the results of process dissociation experiments.

Jacoby's (1991, Experiment 3) exclusion condition participants, in contrast to ours, "were instructed to call an item old only if it was earlier heard. They were warned that the test list would include words that were earlier presented as anagrams and words that were earlier read and were told that those words should be called new" (p. 528). It cannot be ruled out that these differences in instructions led to differences in the memory processes involved in the tasks. In this case, the conclusions of Buchner, Erdfelder, et al. (1997) would hold for a limited range of situations, at best. What is more, one could argue that Buchner, Erdfelder, et al.'s instructions encouraged a source monitoring strategy, which led to a violation of the boundary conditions of the process dissociation procedure, just as instructions encouraging a generate-recognize strategy violated certain other boundary conditions (see Jacoby, 1998). ${ }^{1}$ Therefore, we thought it important to replicate our earlier experiment, modeling the instructions in the exclusion condition as closely as possible to Jacoby's (1991) description of the instructions used in his Experiment 3.

\section{EXPERIMENT 1}

As in Buchner, Erdfelder, et al. (1997), parameters representing memory and guessing processes in the process dissociation procedure were estimated on the basis of (1) genuine process dissociation response frequencies or (2) source monitoring response frequencies. If the hypothesis is correct that the instructions used by Buchner, Erdfelder, et al. in their exclusion test condition were too close to source monitoring instructions, and if this triggered retrieval processes that differ from the ones normally engaged in recognition judgments in the process dissociation procedure, then one would expect the memory parameters to differ between the source monitoring and the process dissociation conditions in the present experiment. In contrast, if the retrieval processes engaged do not depend on how explicitly the instructions point to source discrimination aspects, then no differences between the two conditions in the memory parameters would be expected.

Following Buchner, Erdfelder, et al. (1997), we assumed that guessing, as well as the memory processes, plays a role when participants are responding in the test phase. Therefore, we also used the extended measurement model to estimate the parameters representing memory and guessing processes from participants' observed (inclusion and exclusion instructions) and reconstructed (source monitoring instruction) responses. The extended measurement model was originally suggested by Buchner et al. (1995). It is a multinomial processing tree model (Batchelder \& Riefer, 1990; Hu \& Batchelder, 1994; Riefer \& Batchelder, 1988) and comprises parameters representing recollection, familiarity given no recollection, and guessing in the inclusion and exclusion conditions.

In short, in the extended measurement model, it is assumed that an old response to a Phase 1 item in the inclusion condition can be the result of (1) a recollection of an item (the probability of which is represented by parameter $c$ ); (2) a feeling of familiarity in the absence of recollection (parameter $u_{\mathrm{c}-}$ ); and (3) guessing in the absence of recollection and familiarity (parameter $g_{i}$ ). New responses are given to a Phase 1 word in the absence of recollection, familiarity, and guessing old. In contrast, in the exclusion condition, an old response to a Phase 1 item can result from only two cognitive states, that is, (1) from a feeling of familiarity in the absence of recollection (parameter $u_{\mathrm{c}-}$ ) and (2) from guessing in the absence of recollection and familiarity (parameter $g_{\mathrm{e}}$ ). A new response to a Phase 1 item is given in the exclusion condition (1) if a word is recollected as a Phase 1 word (parameter $c$ ) or (2) if it is not recollected, does not appear familiar, or is not guessed to be old. Given certain additional assumptions (for details, see Buchner, Erdfelder, et al., 1997; Buchner et al., 1995), the four model parameters can be estimated from participants' responses to Phase 1 items and to distractors in the inclusion and exclusion conditions. The memory status of Phase 2 items is undefined 

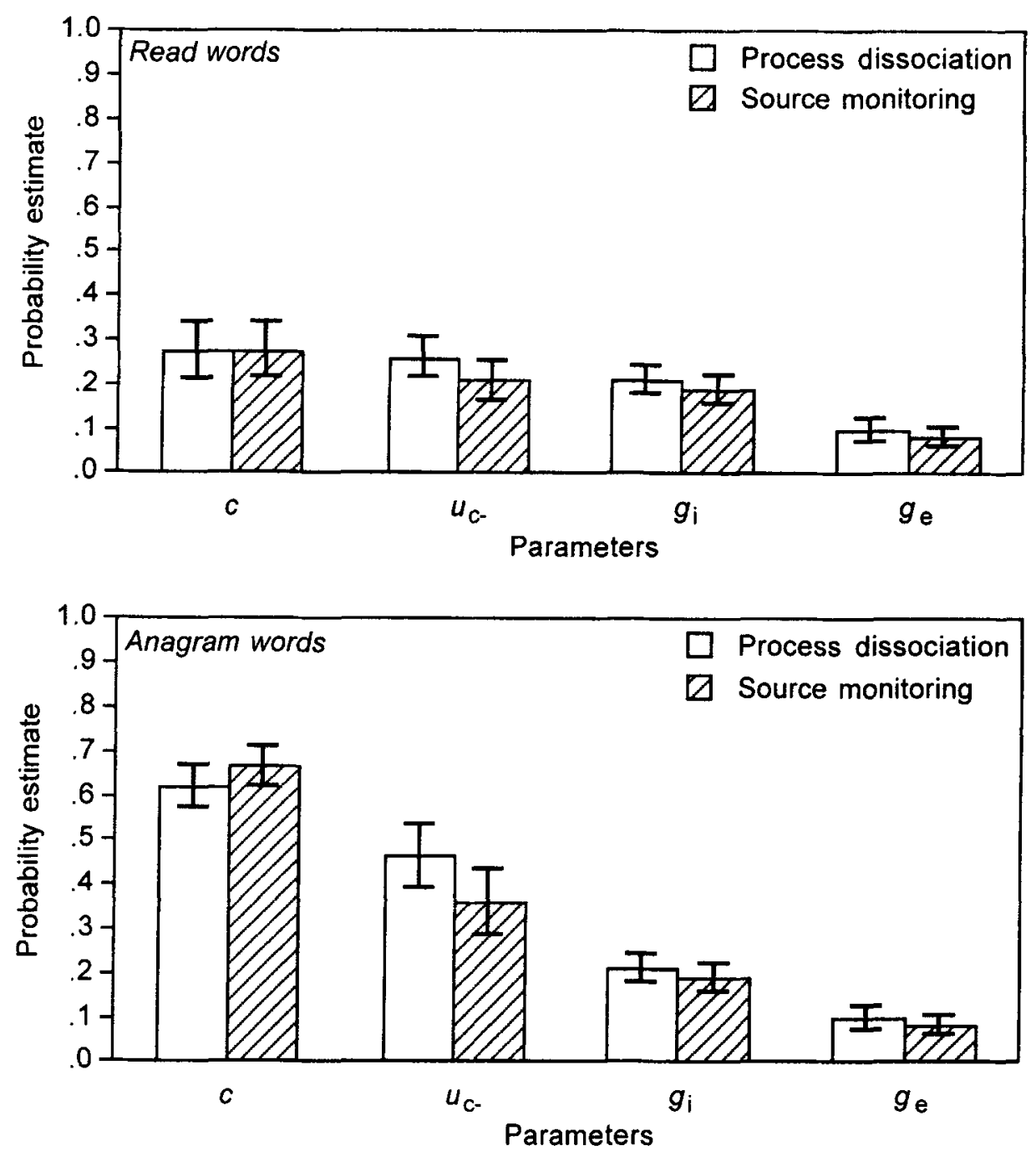

Figure 1. Estimates of parameters representing controlled $(c)$, automatic $\left(u_{c-}\right)$, and guessing processes $\left(g_{\mathrm{i}}\right.$ and $g_{\mathrm{e}}$ ) for the read words (upper panel) and for the anagrams (lower panel) as a function of whether the frequencies underlying the parameter estimates were obtained in process dissociation or source monitoring conditions. The estimates for the guessing parameters are the same for the read words and for the anagrams. The error bars represent $95 \%$ confidence intervals.

within the process dissociation procedure. The purpose of including these items in an experiment is to provide a set of items relative to which Phase 1 items can be included or excluded (Jacoby, 1991). As is typical of process dissociation experiments, responses to Phase 2 items were not analyzed.

\section{Method}

Participants. Participants were 39 female and 25 male students at the University of Trier who either volunteered or received course credit for participating in the experiment. They ranged in age from 19 to 43 years $(M=22.64)$. They were assigned at random to one of the following four experimental groups: the process dissociation inclusion group; the process dissociation exclusion group; the source monitoring inclusion group; and the source monitoring exclusion group.
Stimuli and Apparatus. The stimuli and apparatus were as described in Buchner, Erdfelder, et al. (1997). Very briefly, 100 fiveletter German nouns were assigned to four lists of 25 words each. Words from one of these four lists served as words to be read in Phase 1, words from a second list were presented as anagrams in Phase 1, words from a third list were to be heard in Phase 2, and words from the fourth list were added as distractor words in the recognition memory test in Phase 3 of the experiment. The four lists were rotated through the four presentation conditions (read, anagram, hear, and distractor) such that each set of words was used in each of the presentation conditions.

The anagrams were constructed by keeping the second and fourth letters in each word in their proper positions while rearranging the remaining letters. Words to be heard were recorded and digitized individually on an Apple Macintosh AV computer. During the experiment, the words were presented binaurally through stereo headphones plugged directly into the computer. 
The list of 100 words for the final recognition test was composed of all words from all four word lists. The sequence of words to be read and anagrams to be solved in Phase 1 was randomized for every participant, as was the sequence of words to be heard during Phase 2 and the sequence of test words in Phase 3.

Procedure. The procedure was identical to that described in Buchner, Erdfelder, et al. (1997) except for the exclusion condition instructions. Briefly, in Phase 1 participants read words or solved anagrams presented on the computer screen and wrote the words and the anagram solutions down as fast as possible. The recognition test at the end of the experiment was not mentioned. Participants were allowed a maximum of $30 \mathrm{sec}$ to generate a word from an anagram. After that period, the correct solution was shown and participants wrote it down. In Phase 2, participants heard words, wrote them down, and memorized them for a later recognition test. In Phase 3, each test word was presented on the computer screen for the recognition test. Participants in the process dissociation inclusion group were to respond "old" if they had heard, read, or generated the word from an anagram. They responded "new" if they thought that the word was new.

The major deviation from the Buchner, Erdfelder, et al. (1997) study was that the instructions in the exclusion condition were modeled to point as little as possible to the source monitoring aspect of the task while conforming to the description of the instructions in Jacoby (1991, Experiment 3). Thus, participants in the process dissociation exclusion group were instructed to respond "old" only if they had heard a word in the study phase. They were warned that the test list would also include words they had read or solved as anagrams, and they were told that those words should be called "new," along with the words they had not encountered before. Participants in both source monitoring groups received identical instructions: They were to respond "Phase 1" if they remembered having solved a word as an anagram or having read it in Phase 1 . They were to respond "Phase 2" if they thought they had heard the word in Phase 2. Finally, they were to respond "new" to new words. After the experiment, all participants were debriefed and the purpose of the experiment was described.

Design. The dependent variable was participants' recognition performance. Independent variables were (1) test procedure (process dissociation vs. source monitoring; between subjects) and (2) test condition (inclusion vs. exclusion; between subjects). The inclusion and exclusion "conditions" for the source monitoring participants were created by randomly selecting $50 \%$ of the participants and reconstructing inclusion responses from their source monitoring responses as specified by the mapping rules illustrated in Table 1. The responses of the other half of the participants were used to reconstruct exclusion responses. There were 16 participants in each of the treatment combinations.

Each of the 64 participants contributed 25 responses to the read (or anagram) words and 25 responses to the distractor words, resulting in $N=64 \cdot 50=3,200$ for the model test (see below). We decided that we wanted to detect "small effects" $(w=0.1$ for chisquare tests; $c f$. Cohen, 1977) in the deviations between the actual response probabilities and the response probabilities as predicted by the restricted model defining the null hypothesis. Given $w=.10$, the additional premise that $\alpha=\beta$, and $2 d f$ for the model test, a compromise power analysis (Erdfelder, Faul, \& Buchner, 1996) suggested the choosing of error probabilities of $\alpha=\beta=.0055$ (which implies $\left.\chi_{\text {crit }}^{2}=10.4211\right)^{2}$

\section{Results}

In a first step, we used the unrestricted extended measurement model for the process dissociation procedure (Buchner et al., 1995) to estimate, for the read words, parameter $c$, which is assumed to represent recollection processes; parameter $u_{\mathrm{c}-}$, which is assumed to represent au- tomatic familiarity-related processes; and the guessing parameters $g_{\mathrm{i}}$ and $g_{\mathrm{e}}$. The upper panel of Figure 1 displays these parameter estimates, along with their $95 \%$ confidence intervals. Comparing the parameter estimates based on the process dissociation response frequencies with the estimates based on the frequencies reconstructed from the source monitoring task, we find that both $\hat{c}$ and $\hat{u}_{\mathrm{c}-}$ differ very little between conditions.

In a second step we performed goodness-of-fit tests for a model in which we implemented the equality restrictions that $c$ (process dissociation) $=c$ (source monitoring) and that $u_{\mathrm{c}-}$ (process dissociation) $=u_{\mathrm{c}-}$ (source monitoring). In other words, the hypothesis was tested that the memory processes in the process dissociation conditions do not differ from those in the source monitoring conditions. The two restrictions on the unrestricted model yield $2 d f$ for the model test. The likelihood-ratio goodnessof-fit statistic indicates that the data are compatible with the restricted model $\left[G^{2}(2)=1.79\right],{ }^{3}$

The parameter estimates and $95 \%$ confidence intervals for the anagram data are displayed in the lower panel of Figure 1. They also do not differ much between conditions. As for the read words, we performed goodnessof-fit tests for the model in which we implemented the restrictions that $c$ (process dissociation $)=c($ source $m o n-$ itoring) and that $u_{\mathrm{c}-}$ (process dissociation) $=u_{\mathrm{c}-}$ (source monitoring). The likelihood-ratio goodness-of-fit statistic indicates, again, that the data are compatible with the restricted model $\left[G^{2}(2)=4.62\right]$. Thus, the anagram data replicate the read data.

However, not only the parameter estimates, but also the raw response frequencies regarding Phase 1 items and distractors as obtained in the process dissociation procedure and those reconstructed from the source monitoring design were relatively close (Table 2). ${ }^{4}$ Thus, the entire recognition behavior relevant for the process dissociation procedure seemed to be predictable from source monitoring behavior. We used a chi-square test to test the $H_{0}$ that the process dissociation response frequencies are identical to the response frequencies as derived from source monitoring responses. As in the model-based analysis, responses to Phase 1 words and to distractors were included. This time, however, both read and anagram Phase 1 words were included in the same analysis. The log-likelihood$G^{2}$ for the statistical test of this hypothesis is $G^{2}(6)=$ 10.17 , which is not significant given the critical value $\chi^{2}(d f=6, \alpha=.05)=12.59$. This result is consistent with the substantive hypothesis that neither memory nor judgment processes differed between the process dissociation and source monitoring tasks. The original response frequencies as obtained in the source monitoring conditions are given in Table Al.

\section{Discussion}

The results of the present experiment replicate almost perfectly those reported in Buchner, Erdfelder, et al. (1997). We may therefore reject the hypothesis that the exclusion condition instructions in that study were actu- 
Table 2

Experiment 1: Frequencies of Yes (or Old) and $\mathrm{No}$ (or $\mathrm{New}$ ) Responses to

Phase 1 Words (Read Words and Anagrams), Distractors, and Phase 2 Words

Obtained Directly in the Process Dissociation Condition and Derived From Source Discrimination Data in the Source Monitoring Condition

\begin{tabular}{|c|c|c|c|c|c|c|c|c|}
\hline \multirow[b]{2}{*}{ Test Condition } & \multicolumn{2}{|c|}{ Phase 1, Read } & \multicolumn{2}{|c|}{ Phase 1 , Anagram } & \multicolumn{2}{|c|}{ Distractor } & \multicolumn{2}{|c|}{ Phase 2} \\
\hline & Yes & No & Yes & No & Yes & No & Yes & $\mathrm{No}$ \\
\hline \multicolumn{9}{|c|}{ Process Dissociation Condition } \\
\hline Inclusion & 230 & 170 & 335 & 65 & 84 & 316 & 344 & 56 \\
\hline Exclusion & 96 & 304 & 79 & 321 & 39 & 361 & 306 & 94 \\
\hline \multicolumn{9}{|c|}{ Source Monitoring Condition } \\
\hline Inclusion* & 213 & 187 & 330 & 70 & 75 & 325 & 330 & 70 \\
\hline Exclusion $\dagger$ & 79 & 321 & 55 & 345 & 33 & 367 & 264 & 131 \\
\hline
\end{tabular}

*Phase $l$ and Phase 2 responses were aggregated to get yes responses. 'Phase $l$ and new responses were aggregated to get no responses.

ally so close to source monitoring instructions that they induced retrieval strategies that are typical of source monitoring tasks, but not of process dissociation tasks. Therefore, Jacoby's (1998) argument that certain instructions may lead to a violation of boundary conditions of the process dissociation procedure does not apply to the present context.

As a consequence, the present experiment reaffirms the conclusion reached by Buchner, Erdfelder, et al. (1997) that the memory processes involved in recognition judgments in the process dissociation procedure are the same as those involved in standard source monitoring tasks. In addition, the present results corroborate the idea that recognition judgments in the process dissociation procedure may be considered redundant given standard source monitoring instructions. This is so because process dissociation responses, as well as estimates of parameters representing memory processes, can be recovered without loss from source monitoring data. Carrying out a source monitoring procedure and subsequently recovering process dissociation parameters can have a major advantage: If participants in the process dissociation exclusion condition can be expected to have difficulty following process dissociation instructions (see Graf \& Komatsu, 1994), the process dissociation instructions can be replaced with the simpler and easier to follow source monitoring instructions. This may be especially important with certain samples (e.g., small children).

\section{EXPERIMENT 2}

Experiment 2 was designed to test the hypothesis that the same memory processes are involved in fame judgments in a source monitoring and a process dissociation task. The reasoning that source monitoring processes are involved in fame judgments was already brought forward by Dodson and Johnson (1996). It has, to our knowledge, not yet been tested empirically.

In a typical fame judgment task (e.g., Jacoby, Kelley, Brown, \& Jasechko, 1989), participants first read a list of nonfamous names in an incidental learning situation. In a second phase, participants judge whether each of a list of famous and nonfamous names belongs to a famous or a nonfamous person. Usually, precautions are taken in order to keep recollection of the study phase names low (see Jacoby, Woloshyn, \& Kelley, 1989). (Implicit) memory for previously seen names is said to be demonstrated if old nonfamous names are more likely to be called famous than new nonfamous names. This phenomenon is referred to as the false fame effect (Jacoby, Woloshyn, \& Kelley, 1989). The problem, of course, is that not only familiarity-based but also recollective processes may influence these fame judgments. This has stimulated the use of the process dissociation procedure in an attempt to decompose fame judgments into controlled recollections and automatic familiarity-based processes in much the same way as has been attempted with recognition judgments (e.g., Buchner \& Wippich, 1996; Jennings \& Jacoby, 1993).

In order to be able to do so, an inclusion and an exclusion condition must be defined for the fame judgment task. In the typical inclusion condition, participants are misinformed that all the names from the study list were the names of famous people, so that each name recognized as a study phase name needs to be rated "famous." In this condition, controlled recollection as well as automatic familiarity-based memory processes should lead to famous judgments. In the exclusion condition, participants are correctly informed that all names from the study list were names of nonfamous people, so that if they recognize a name as one that was presented in the study phase, they need to "exclude" it and judge it "nonfamous." It is assumed that famous judgments to study phase names in the exclusion condition result from automatic memory processes, which are not counteracted by controlled recollective processes (Jennings \& Jacoby, 1993).

In parallel to our reasoning with regard to recognition judgments, the processes that lead to fame judgments in the process dissociation procedure may be described differently. First of all, as with recognition judgments, there 
are two sources of "familiarized" (in some sense "old") items. Names may be (1) nonfamous names with which participants were familiarized during the study phase (Source A) or (2) names belonging to famous persons with which participants were familiarized prior to the experiment (Source B; e.g., they may have read a given name a number of times in the newspapers). In addition, there are new, nonfamous names just as there are new items in a recognition task. As an alternative to the automatic memory process proposed by Jacoby and co-workers, a process of source confusion could be responsible for participants' arriving at a famous response to a study phase name. Participants may recollect very well that they have read that name before, but they simply cannot remember where. In some of these situations, they will decide to respond "famous" to such names, even if the name was a nonfamous name from the study phase. This hardly qualifies as a "clear example of memory without awareness" (Dywan, Segalowitz, Henderson, \& Jacoby, 1993, p. 39), but rather is simple source confusion. At this point, we have reached the same conclusion for fame judgments as Buchner, Erdfelder, et al. (1997) did for recognition judgments. It should, therefore, be possible to reconstruct fame judgments in a process dissociation procedure from appropriate source monitoring judgments.

The right half of Table 1 illustrates how this is done. It shows the relation between famous and nonfamous responses in the process dissociation procedure and study phase, famous, and nonfamous responses in a source monitoring task in which participants are instructed to distinguish between the mutually exclusive categories of study phase names, new famous names, and new nonfamous names. Participants in all test conditions will respond "famous" to a name if they rate it to be the name of a famous person (row 2 in Table 1 ). If they think it is neither a famous name nor a name that was presented in the experiment before, they will respond nonfamous in all three conditions (row 3 in Table 1). The responses differ between test conditions, however, with regard to names that participants consider to be from the study phase. Following instructions, in the source monitoring condition, participants will respond "study phase" in these cases; in the process dissociation inclusion condition, they will respond "famous"; and in the process dissociation exclusion condition, they will respond "nonfamous." Thus, we may assume that participants in the source monitoring condition would have responded "famous" instead of "study phase" had they been given inclusion test instructions, and they would have responded "nonfamous" instead of "study phase" had they been given exclusion test instructions.

Experiment 2 was basically modeled after Experiment 1 of Jennings and Jacoby (1993). That is, in the study phase, participants read aloud a list of names presented one after the other on the computer screen under divided attention conditions. They were led to believe, however, that their main task was to find sequences of three odd numbers in a row in a continuous string of digits presented auditorily.

\section{Method}

Participants. Participants were 88 students at the University of Trier who either volunteered or received course credit for participating in the experiment. An equal number of them was assigned at random to the source monitoring inclusion group, the source monitoring exclusion group, the process dissociation inclusion group, and the process dissociation exclusion group.

Two sets of names were used, Set A and Set B. Due to an error of the experimenters, the sets were not distributed equally between groups. In order to balance name sets, 8 participants were excluded from all data analyses at random, but with the restriction that each of the experimental groups should end up with 20 participants, half of whom had been assigned Set A during study and half Set B. These participants ranged in age from 19 to 41 years $(M=22.38$, $S D=3.91$ ), and 52 of them were female.

Stimuli and Apparatus. Lists A and B each comprised 40 nonfamous names. Both of these lists were used at test, and for a given participant, one of the lists was presented in the study phase. Additionally, 20 nonfamous names were used as filler and buffer items in the study phase. The list of famous names comprised 82 names; 41 of them were men's and 41 were women's. These names were chosen from a pool of 725 names of famous people who had achieved fame in different fields such as sports, arts, or politics. In a norming study, 54 participants rated the fame of these names on a 5-point scale. An average rating of 3 indicated that the name seemed familiar to the participants, but they did not know what the respective person had done to achieve fame. The average fame rating was $M=$ $3.17(S D=.20)$ for male and $M=2.88(S D=.60)$ for female names. Examples are Robert Altman, Anatoli Karpow, Gertrude Stein, and Vivien Leigh. In addition, 10 names of very famous women and men were selected. Five of them were presented at the beginning and five at the end of the study list. They served as primacy and recency buffers and were to convince participants in the inclusion condition that there had in fact been famous names in the study list.

Eighty nonfamous names were chosen from phone books or made up, paralleling 80 of the 82 famous names in the following characteristics: number of letters in the first names and surnames, number of first names and surnames beginning with a given letter, and ethnicity of names. They were randomly divided into two sets, $A$ and $B$. An additional 10 nonfamous names were used as fillers in the study lists. Thus, a study list consisted of 60 names: 10 very famous names at the beginning and end, 10 filler names, and 40 names from either Set A or Set B. Half of the participants in each condition were assigned to Set $A$ and half to Set $B$.

The test list consisted of the 40 nonfamous names from Set $A$, the 40 nonfamous names from Set $B$, and the 82 famous names. Thus, slightly more than half of the names in the test list belonged to famous people, and participants were told that "more than half of the names presented belong to famous people." This was done in order to induce a liberal response bias: Participants in pilot studies had demonstrated a very conservative response bias, hardly ever judging study phase names or new names as famous.

Two sequences of single-digit numbers were constructed for the distractor task, one of length 40 , for a practice session, and one of length 80 , for the study phase. The sequence in the practice session contained 6 runs of three uneven numbers in a row, and the sequence in the study phase contained 12 such runs. Between 1 and 5 digits were presented between runs.

Procedure. Participants were told that their main task was to detect runs of digits. In an attempt to make this cover story more con- 
Table 3

Experiment 2: Frequencies of Yes and $N o$ Responses to Nonfamous Study Phase Names, New Nonfamous Names, and New Famous Names Obtained Directly in the Process Dissociation Condition and Derived From Source Discrimination Data in the Source Monitoring Condition

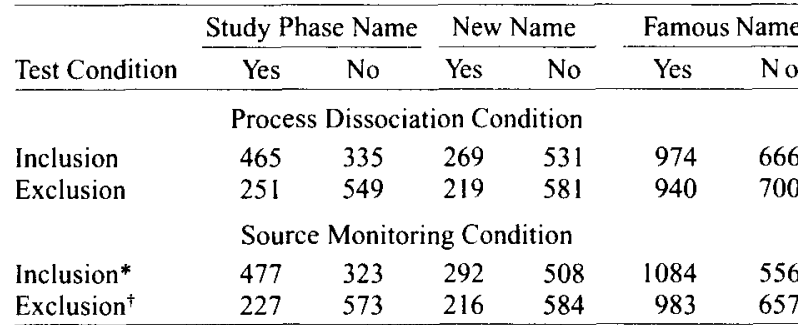

* Study phase and famous responses were aggregated to get yes responses. $\dagger$ Study phase and no responses were aggregated to get no responses.

vincing, a practice session was devoted to this task. On the computer screen of an Apple Macintosh Power Book $165 \mathrm{c}$, participants received the instruction that they were to press a horn handed to them in advance whenever they detected a set of three odd numbers in a row in an auditorily presented continuous stream of digits. An example $(3,9,5)$ was given. Prerecorded digits were then presented in a male voice with a rate of 1 digit per $1.5 \mathrm{sec}$.

After the practice session, participants were instructed that a distractor task would be added to their main task. They were told to read aloud the names presented one after the other on the computer screen. The instructions were modeled after those given by Jennings and Jacoby (1993) and stressed that participants should concentrate on the detection task and devote as little attention as possible to their pronunciation of names. Additionally, the instructions prepared the participants for the experimenter's saying "miss" whenever there was a miss of a 3-odd-digit sequence and "no" for each false alarm. Presentation of the digits was identical to that in the practice session. The experimenter kept track of the misses and false alarms. A name was presented on the screen at a rate of 1 per $2 \mathrm{sec}$ in a random order for each participant, except for the primacy and recency buffer names, which were presented in a fixed order

In the test phase, participants in the source monitoring condition were told to decide, for each name, whether it had appeared in the beginning of the experiment, belonged to a famous person, or whether they didn't know the name, by clicking, with the computer mouse, on the Beginning, Famous, or No screen buttons, respectively. They were informed that each name presented could be categorized unambiguously. Categorization was unambiguous because ambiguous names (i.e., famous names from the beginning of the experiment) were not presented in the test phase. Participants in the process dissociation conditions were told to decide, for each name, whether it belonged to a famous person or not, and to press the appropriate screen button. It was mentioned that some of the names had just been read. In the inclusion condition, participants were told that all previously presented names belonged to famous persons, so that if they recognized a name, they could be sure it belonged to a famous person. In contrast, in the exclusion condition, participants were told that all previously read names that appeared in the test phase belonged to nonfamous people, so that if they recognized a name, they could be sure it did not belong to a famous person. To induce a liberal response bias, all participants were informed that the majority of the names belonged to famous people, that these people were not as famous as, for example, Helmut Kohl, and that we would not ask them further questions about the names they judged as belonging to famous people (see Jennings \& Jacoby, 1993).
Each name was then presented in the center of the computer screen in an individual random order for each participant, and the appropriate response buttons appeared below it. These buttons were labeled "yes" and "no" in the process dissociation conditions and "beginning," "famous," and "no" in the source monitoring condition. Presentation of names was self-paced. After a judgment had been made about a name, that name disappeared, and a button labeled "next name" appeared. Clicking this button initiated the presentation of the next name. This was done in order to minimize participants' tendency to repeatedly click the same response button.

Every 15 trials, participants received feedback about the number of famous names they had already detected. Again, this was done to help participants maintain a liberal response bias. In the inclusion condition, this feedback was incorrect in that the famous names rated famous and the study phase names rated famous were aggregated and presented as famous names that had been detected, because participants had been led to believe that study phase names were famous. Additionally, the instructions were summarized again on the feedback screen for all participants, and the number of names remaining to be judged was indicated. After the experiment, all participants were debriefed.

Design. The dependent variable was the participants' fame judgment performance. Independent variables were (1) test procedure (process dissociation vs. source monitoring; between subjects) and (2) test condition (inclusion vs. exclusion; between subjects). There were 20 participants in each of the treatment combinations of this $2 \times 2$ design. The inclusion and exclusion "conditions" for the source monitoring participants were created by randomly selecting $50 \%$ of the participants and reconstructing inclusion responses from their source monitoring responses as specified by the mapping rules illustrated in the right half of Table 1 . The responses of the other half of the participants were used to reconstruct exclusion responses.

Each of the 80 participants responded to 40 old nonfamous names and to 40 new nonfamous names, resulting in $N=80 \cdot 80=$ 6,400 for the model test. Given $2 d f$ for the model test, a compromise power analysis showed that in order to detect "small effects" of $w=0.1$ for chi-square tests in the deviations between the actual response probabilities and the response probabilities as predicted by the restricted model defining the null hypothesis, error probabilities of $\alpha=\beta=.0001$ (which implies $\chi_{c r i t}^{2}=18.72$ ) should be chosen. Note, however, that the same conclusions would have been drawn had we chosen $\alpha=.05$.

\section{Results}

On average, participants missed $1.92(S D=1.87)$ of the 12 sets of odd numbers in the distractor task, and they produced $.89(S D=1.21)$ false alarms. These data did not vary as a function of test condition [both $F \mathrm{~s}(3,76) \leq 1$ ].

Table 3 shows the frequencies of famous (yes responses) and nonfamous (no responses) judgments for nonfamous study phase names, new nonfamous names, and famous names for the process dissociation groups. For the source monitoring groups, Table 3 shows these frequencies as reconstructed from the source monitoring data according to the mapping rules illustrated in Table 1. The original source monitoring frequency data are presented in Table A2 in the Appendix.

Figure 2 displays the parameter estimates for the recollection parameter $c$, the familiarity parameter $u_{\mathrm{c}-}$, and the guessing parameters $g_{\mathrm{i}}$ and $g_{\mathrm{e}}$, together with their $95 \%$ confidence intervals. The confidence intervals for parameter $\hat{c}$-representing recollection-in the process 


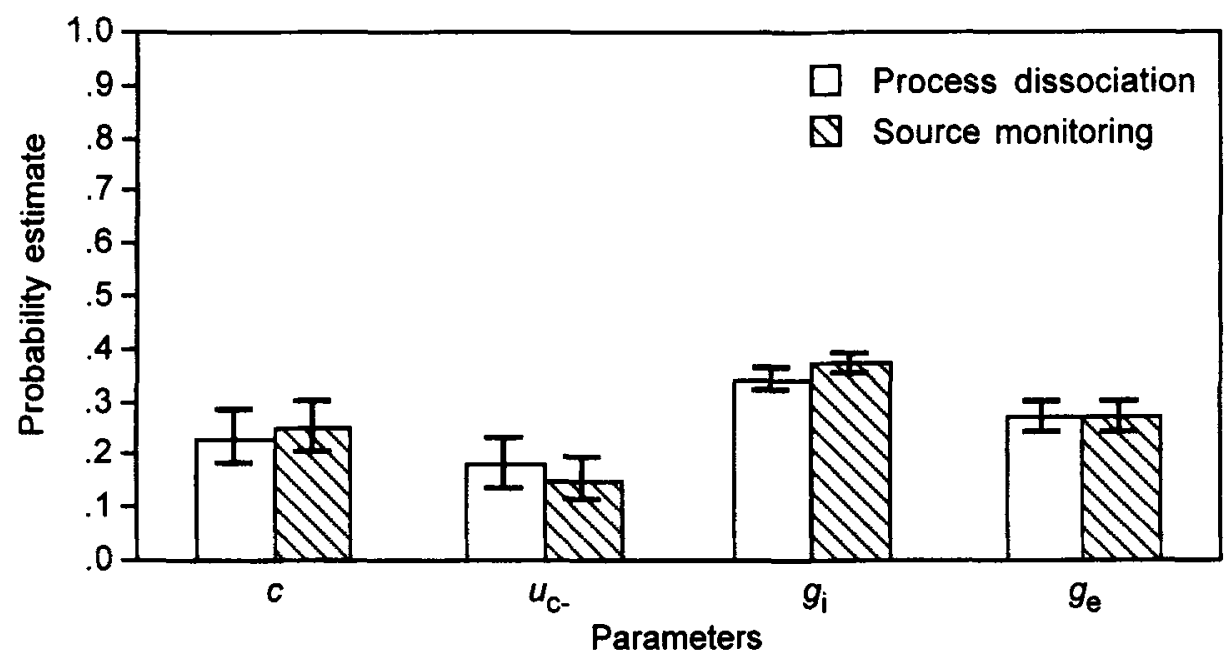

Figure 2. Estimates of parameters representing controlled (c), automatic $\left(u_{c-}\right)$, and guessing processes $\left(g_{\mathrm{i}}\right.$ and $\left.g_{\mathrm{e}}\right)$ derived from the responses to old and new nonfamous names as a function of whether the frequencies underlying the parameter estimates were obtained in process dissociation or source monitoring conditions. The error bars represent $95 \%$ confidence intervals.

dissociation and in the source monitoring condition overlap almost completely, showing that these parameter estimates differ very little between conditions. The same is true for parameter $\hat{u}_{\mathrm{c}-}$, representing familiarity. We performed goodness-of-fit tests for the model in which the parameters representing the controlled recollections as well as those representing the automatic, familiaritybased memory processes in the process dissociation condition were set equal to those in the source monitoring condition. More precisely, we imposed on the model the restrictions that $c$ (process dissociation) $=c$ (source monitoring) and that $u_{\mathrm{c}-}$ (process dissociation) $=u_{\mathrm{c}-}$ (source monitoring). The likelihood-ratio goodness-of-fit statistic indicates that the data are very compatible with the restricted model $\left[G^{2}(2)=1.06\right]$.

According to our original hypothesis, response strategies may or may not be equivalent in the current implementation of the process dissociation procedure and the source monitoring task. However, the response frequencies displayed in Table 3 make it quite obvious that there is very little difference between the responses to study phase names and new names obtained in the process dissociation procedure and those reconstructed from the source monitoring data. We thus tested the even stronger hypothesis that there is no difference between the memory and judgment processes in the two tasks. The $H_{0}$ that the response frequencies obtained in the original process dissociation conditions are identical to those reconstructed from the source monitoring responses was tested using a chi-square test. As in the model-based analysis, responses to study phase names and to new names were included. The log-likelihood $G^{2}$ for the statistical test of this hypothesis is $G^{2}(4)=3.57$, which is not significant given the critical value $\chi^{2}(d f=4, \alpha=.05)=9.49$. Consequently, the substantive hypothesis that both memory and judgment processes do not differ between the process dissociation and source monitoring fame judgment tasks need not be rejected. Besides its relevance with regard to response strategies, the result of this test adds force to our line of reasoning because this result does not depend on the specific model used to derive the measures for the memory and judgment processes. In short, Experiment 2, using a fame judgment task, extended the results of Experiment 1 , as hypothesized.

\section{GENERAL DISCUSSION}

Experiment 1 showed that Buchner, Erdfelder, et al.'s (1997) results need not be called into question on the basis of the instructions that were given. Those instructions could have been conceived of as closer to typical source monitoring instructions than Jacoby's (1991) instructions. With instructions modeled after Jacoby's (1991), however, our earlier results were closely replicated. Thus, independent of the instructions given, there is close correspondence between participants' recognition judgments in the process dissociation procedure and in source monitoring tasks. This finding is compatible with the conclusion that memory and judgment processes in a process dissociation recognition experiment do not differ from memory and judgment processes obtained in a source monitoring experiment.

The goal of Experiment 2 was to extend further the generality of this conclusion by using fame judgments rather than recognition judgments. The results show that the original process dissociation fame judgments are predictable from the process dissociation responses as reconstructed from source monitoring fame judgments. This is compatible with the hypothesis that both the memory and the guessing processes underlying fame judgments do 
not differ between the process dissociation and the source monitoring task. We thus expect that for typical recognition and false fame experiments, a source monitoring and a process dissociation procedure will yield similar results.

In our model-based analyses, we allowed the guessing parameters to differ between the source monitoring and process dissociation conditions. We reasoned that guessing biases may be affected by procedural differences between conditions (see Buchner et al., 1995). For instance, the presence of three screen buttons (source monitoring) instead of two (process dissociation) may induce participants to assume different base rates and thus may provoke different biases. If indeed guessing processes had differed between source monitoring and process dissociation conditions, we still would have been able to conclude that memory processes do not vary as a function of the task. In the present case at least, the analyses showed that even the raw frequencies of responses toward Phase 1 items (study phase names) and distractors (new names) were identical, so that we may conclude that guessing biases did not differ. Therefore, no matter which model is used, the conclusion is that responses toward Phase 1 items (study phase names) and distractors (new names) are identical in the source monitoring and process dissociation conditions.

Our results converge with newer results of other authors. For example, Hintzman, Caulton, and Levitin (1998) found that parameters describing the retrieval dynamics in an exclusion task (Experiment 1) almost perfectly agreed with those obtained in a source monitoring task (Experiment 2). Electrophysiological evidence on the similarity of source discrimination processes and conscious recollection processes was recently reported by Rugg, Schloerscheidt, and Mark (1998). Using eventrelated potentials, they found that the same brain regions appear to be involved in correct source judgments (as obtained in a source monitoring task) and correct remember judgments (as obtained in a remember-know task).

What do our findings, along with those of others, imply with regard to the interpretation of the process dissociation memory parameters? For illustration, let us take a closer look at the recollection parameter $c$ in process dissociation measurement models. One may assume that $c$ captures all conscious memory processes (see Jacoby, Yonelinas, \& Jennings, 1997). If this conceptualization were reasonable, then $c$ should not be affected by manipulations such as list similarity in a recognition task; but it is (see Mulligan \& Hirshman, 1997). Consequently, Mulligan and Hirshman have argued that the recollection parameter represents "recollection that is diagnostic of list source" (1997, p. 294). Similarly, McElree, Dolan, and Jacoby $(1999$, p. 565$)$ used the process dissociation procedure "to place familiarity information and source information in opposition." Does this mean that recollection, as measured by the process dissociation model, is actually source discrimination? In general, the answer is no. However, the two can be closely related if specific as- sumptions are made, as we discuss in the following paragraphs.

In source monitoring models (Batchelder \& Riefer, 1990 ), there is one parameter for item memory and one for memory of the source from which the item stems. In these models, the source memory parameter is conditional upon item memory: Naturally, one can remember the source from which an item stems only if one remembers the item itself. Borrowing terms from the process dissociation literature, one may say that item memory is redundant with respect to source memory. In process dissociation models, the recollection parameter is supposed to capture controlled memory processes. The nature of the relation between recollection and familiarity is not as obvious as that between source and item memory. Whether redundancy, independence, or exclusivity should be assumed has been discussed extensively (see Buchner et al., 1995; Curran \& Hintzman, 1995; Jacoby, Yonelinas, \& Jennings, 1997).

If independence of recollection and familiarity is assumed-an assumption that Jacoby and colleagues have vigorously defended (e.g., Jacoby, Yonelinas, \& Jennings, 1997) - an item that is recollected can either seem familiar, or not. If, instead, and in parallel to the source monitoring model, a redundancy assumption is made, recollection always implies a feeling of familiarity. Buchner, Erdfelder, et al. (1997) showed analytically that, if such a redundancy assumption is made (and guessing does not play a role), the process dissociation recollection parameter is indeed identical to the source memory parameter of Batchelder and Riefer's (1990) model. However, under the independence assumption favored by Jacoby and colleagues, the recollection parameter in a process dissociation model is a complicated function of item memory, source memory, and possible guessing processes. In other words, recollection as measured by the independence model cannot be equated with source memory.

Jacoby (1999, pp. 12-14) agreed with this conclusion. However, he advocated using the independence measurement model of the process dissociation procedure rather than a source monitoring measurement model because "reliance on the source-monitoring model required more parameters to fit the results" of his experiments (p. 15). Using a source monitoring model, he observed age effects on both the item detection and the source discrimination parameter. In contrast, using an independence measurement model, he observed age effects on recollection, but not on familiarity.

Jacoby (1999, p. 15) consequently interpreted that "the distinction between recollection and familiarity is more fundamental than is the distinction between recognition and source memory." We do not believe that his results support this interpretation. In particular, they do not prove that the process dissociation model is superior to the source monitoring model. First, his results conform to prior research on aging and source monitoring: Both item and source memory decrease with age (e.g., Bayen \& 
Murnane, 1996; Henkel, Johnson, \& DeLeonardis, 1998; Light, LaVoie, Valencia-Laver, Albertson Owens, \& Mead, 1992). Thus, there is simply no reason to expect invariance of item and/or source memory throughout age groups. Second, the fact that a given model requires fewer parameters than an alternative model is not a strong argument in favor of that model, especially when these "fewer" parameters have been shown to be inadequately affected by certain manipulations (cf. Mulligan \& Hirshman, 1997). Therefore, Jacoby's findings of invariance do not help in identifying the best measurement model.

We maintain that viewing process dissociation judgments as special cases of source monitoring judgments can be advantageous for the following reasons: (1) the source monitoring procedure is easier for participants to understand than is the process dissociation procedure, and (2) the source monitoring parameters "have clear-cut psychological interpretations because they are integrated into a network of psychological hypotheses and theories" (Buchner, Erdfelder, et al., 1997, p. 516).

Buchner, Erdfelder, et al. (1997, p. 516) asserted at the end of their article that "applications of Jacoby's (1991) procedure to other [than recognition] judgments proved to be very successful, and currently we can see no reason to replace these applications by other types of memory tasks." The generality of that conclusion is called into question by our new findings and should thus be modified. On the one hand, we still cannot see how Stroop tasks (Lindsay \& Jacoby, 1994) or, for that matter, other tasks in which response speed is crucial for dissociating automatic from controlled processes can be reduced to source monitoring tasks (see Spieler, Balota, \& Faust, 1996, however, for difficulties arising with these tasks). On the other hand, given source monitoring tasks, the process dissociation procedure is redundant not only for recognition judgments but also for fame judgments. Given that others have shown the process dissociation parameters to be inappropriately affected by experimental manipulations of source similarity (see Mulligan \& Hirshman, 1997), we suggest that standard source monitoring tasks be used instead of recognition and false fame tasks in the process dissociation procedure.

\section{REFERENCES}

Anooshian, L. J., \& Seibert, P. S. (1996). Conscious and unconscious retrieval in picture recognition: A framework for exploring gender differences. Journal of Personality \& Social Psychology, 70, 637645

Batchelder, W. H., \& Riefer, D. M. (1990). Multinomial processing models of source monitoring. Psychological Review, 97, 548-564.

BAyEN, U. J., \& Murnane, K. (1996). Aging and the use of perceptual and temporal information in source memory tasks. Psychology \& Aging, 11, 293-303

Begg, 1. M., Anas, A., \& Farinacci, S. (1992). Dissociation of processes in belief: Source recollection, statement familiarity, and the illusion of truth. Journal of Experimental Psychology: General. 121, 446-458.

Buchner, A., Erdfelder, E. Stefeens, M. C. \& Martensen. H. (1997). The nature of memory processes underlying recognition judgments in the process dissociation procedure. Memory \& Cognition, 25, 508-517.

Buchner, A., Erdfelder, E., \& Vaterrodt-PlünNecke, B. (1995) Toward unbiased measurement of conscious and unconscious memory processes within the process dissociation framework. Journal of Experimental Psychology: General, 124, 137-160.

Buchner, A., Faul, F., \& Erdfelder, E. (1996). G•Power: A priori, post-hoc, and compromise power analyses for the Macintosh (Version 2.1.1) [Computer program]. Trier, Germany: University of Trier. Available: URL: http://www.psychologie.uni-trier.de:8000/projects/ gpower.html

Buchner, A., Steffens, M. C., Erdfelder, E., \& Rothkegel, R. (1997). A multinomial model to assess fluency and recollection in a sequence learning task. Quarterly Journal of Experimental Psychology, 50A, 631-663.

BUCHNER, A., \& WiPPICH, W. (1996). Unconscious gender bias in fame judgments? Consciousness \& Cognition, 5, 197-220.

COHEN, J. (1977). Statistical power analysis for the behavioral sciences (Rev. ed.) Hilisdale, Ni: Erlbaum.

Cowan, N., \& Stanler, M. A. (1996), Estimating unconscious processes: Implications of a general class of models. Journal of Experimental Psychology: General, 125, 195-200.

Curran, T., \& HinTzMaN, D. L. (1995). Violations of the independence assumption in process dissociation. Journal of Experimental Psychology: Learning, Memory, \& Cognition, 21, 531-547.

Dehn, D. M., \& ENGELKamp, J. (1997). Process dissociation procedure: Double dissociations following divided attention and speeded responding. Quarterly Journal of Experimental Psvchologv, 50A, 318-336.

Dodson, C. S., \& Johnson. M. K. (1996). Some problems with the process-dissociation approach to memory. Journal of Experimental Psychology: General, 125, 181-194.

Dywan, J., Segalowitz, S. J., Henderson, D., \& Jacoby, L. L. (1993). Memory for source after traumatic brain injury. Brain \& Cognition. 21, 20-43.

ERDFELDER, E, \& BUCHNER, A. (1998). PJocess-dissociation measurement models: Threshold theory or detection theory? Joumal of Experimental Psychology: General, 127, 83-96.

Erdfelder, E., FAul, F., \& Buchner, A. (1996). GPower: A general power analysis program. Behavior Research Methods, Instruments, \& Computers, 28, 1-11.

Graf, P., \& Komatsu, S.-I. (1994). Process dissociation procedure: Handle with caution! European Journal of Cognitive Psychologv, 6, 113-129.

Henkel, L. A., Johnson, M. K., \& DeLeonardis, D. M. (1998). Aging and source monitoring: Cognitive processes and neuropsychological correlates. Journal of Experimental Psychologv: General, 127, 251268.

Hintzman, D. L., Caulton, D. A., \& Levitin, D. J. (1998). Retrieval dynamics in recognition and list discrimination: Further evidence of separate processes of familiarity and recall. Memory \& Cognition. 26, $449-462$

HU, X., \& BATCHELDER, W. H. (1994). The statistical analysis of general processing tree models with the EM algorithm. Psychometrika. 59, $21-48$.

JACOBY, L. L. (1991). A process dissociation framework: Separating automatic from intentional uses of memory. Journal of Memory \& Language, 30, 513-541.

JACOBY, L. L. (1998). Invariance in automatic influences of memory: Toward a user's guide for the process-dissociation procedure. Journal of Experimental Psychology: Learning, Memory. \& Cognition, 24, 3 26.

JACOBY, L. L. (1999). Ironic effects of repetition: Measuring age-related differences in memory. Journal of Experimental Psuchology: Learning, Memory, \& Cognition, 25, 3-22.

J ACOBY, L. L., BEGG, I. M. \& TOTH, J. P. (1997). In defense of functional independence: Violations of assumptions underlying the processdissociation procedure? Journal of Experimental Psychology: Learning, Memory, \& Cognition, 23, 484-495.

JaCOBy, L. L., Kelley, C., Brown. J., \& Jasechko, J. (1989). Becoming famous overnight: Limits on the ability to avoid unconscious in- 
fluences of the past. Journal of Personality \& Social Psychology, 56 326-338.

JaCOBY, L. L., Woloshy , V., \& Kelley, C. (1989). Becoming famous without being recognized: Unconscious influences of memory produced by dividing attention. Journal of Experimental Psychology: General, 118, 115-125.

Jacoby, L. L., Yonelinas, A. P., \& Jennings, J. M. (1997). The relation between conscious and unconscious (automatic) influences: A declaration of independence. In J. Cohen \& J. W. Schooler (Eds.), Scientific approaches to the question of consciousness (pp. 13-47). Hillsdale, NJ: Erlbaum.

JENNingS, J. M., \& JACOBY, L. L. (1993). Automatic versus intentional uses of memory: Aging, attention, and control. Psychology \& Aging, 8, 283-293.

Johnson, M. K., Hashtroudi, S., \& Lindsay, D. S. (1993). Source monitoring. Psychological Bulletin, 114, 3-28.

Komatsu, S.-I., Graf, P., \& UTTL. B. (1995). Process-dissociation procedure: Core assumptions fail, sometimes. European Journal of $\mathrm{Cog}$ nitive Psychology, 7, 19.40.

LECOMPTE, D. C. (1995). Recollective experience in the revelation effect: Separating the contributions of recollection and familiarity Memory \& Cognition, 23, 324-334.

Light, L. L., La Voie, D., Valencia-Laver, D., Albertson OWens, S. A., \& MEAD, G. (1992). Direct and indirect measures of memory for modality in young and older adults. Journal of Experimental Psychology: Learning, Memory, \& Cognition, 18, 1284-1297.

LINDSAY, D. S.. \& JACOBY, L. L. (1994). Stroop process dissociations: The relationship between facilitation and interference. Journal of Experimental Psvchology: Human Perception \& Performance, 20, 21 9-234.

MCBRide, D. M., \& Dosher. B. A. (1999), Forgetting rates are comparable in conscious and automatic memory: A process-dissociation study. Journal of Experimental Psychology: Learning, Memory, \& Cognition, 25, 583-607.

MCELrfe, B., DOLAN, P. O.. \& JaCOBY, L. L. (1999). Isolating the contributions of familiarity and source information to item recognition: A time course analysis. Journal of Experimental Psychology: Learning. Memorv, \& Cognition, 25, 563-582.

Mulligan, N. W., \& Hirshman, E. (1997). Measuring the bases of recognition memory: An investigation of the process-dissociation framework. Journal of Experimental Psvchology: Learning, Memorv, \& Cognition, 23, 280-304

NeAl. A., Hesketh, B., \& ANDREws. S. (1995). Instance-based categorization: Automatic versus intentional forms of retrieval. Memory \& Cognition, 23, 227-242

Nelson, D. L., BennetT, D. J., \& XU. J. (1997). Recollective and automatic uses of memory. Journal of Experimental Psychology: Learning, Memory, \& Cognition, 23, 872-885

Riefer, D. M., \& BATChEL.DER, W. H. (1988). Multinomial modeling and the measurement of cognitive processes. Psychological Review, 95, 318-339.

Riefer, D. M., \& BATCHELDER, W. H. (1991). Statistical inference for multinomial tree models. In J.-P. Doignon \& J.-C. Falmagne (Eds.), Mathematical psvchologv: Current developments (pp. 313-335). New York: Springer-Verlag.
Roediger, H. L., III, \& MCDermott, K. B. ( 1994). The problem of differing false-alarm rates for the process dissociation procedure: Comment on Verfaellie and Treadwell (1993). Neuropsychology, 8, 284-288.

RothKegeL, R. (1996). Apple tree [Computer program]. Trier, Germany: University of Trier. Available: URL: http://www.psychologie.uni-trier.de:8000/projects/AppleTree.html

Rugg, M. D., Schloerscheidt, A. M., \& MARK, R. E. (1998). An electrophysiological comparison of two indices of recollection. Journal of Memory \& Language, 39, 47-69.

Spieler, D. H., Balota, D. A., \& Faust, M. E. (1996). Stroop performance in healthy younger and older adults and in individuals with dementia of the Alzheimer's type. Journal of Experimental Psychology: Human Perception \& Performance, 22, 461-479.

Toth. J. P., REINGOLD, E. M., \& JaCOBY, L. L. (1994). Toward a redefinition of implicit memory: Process dissociations following elaborative processing and self-generation. Journal of Experimental Psychology: Learning. Memory, \& Cognition, 20, 290-303.

YONELINAS, A. P., \& JACOBY, L. L. (1995). Dissociating automatic and controlled processes in a memory-search task: Beyond implicit memory. Psychological Research, 57, 156-165

\section{NOTES}

1. In addition, different instructions could lead participants to adopt different response strategies. This would not be a problem for Buchner, Erdfelder, et al. (1997) because we postulated only that the memory processes are the same in recognition judgments in the process dissociation procedure and in source monitoring tasks. Response strategies should not affect memory processes, but only guessing processes, which are represented by separate guessing parameters in the measurement model that was used (see Buchner et al., 1995).

2 . The power calculations were conducted using the G・Power program (Buchner, Faul, \& Erdfelder, 1996). The standard assumptions of multinomial sampling (i.e., independent observations drawn from the same multinomial distribution) enter into our power calculations. These assumptions may be called into question because the raw data were aggregated across participants and items. However, Riefer and Batchelder (1991) suggested that the $G^{2}$ goodness-of-fit test is rather robust against modest violations of these assumptions. Because both our participants and items are rather homogeneous samples, we think it is appropriate to use our power calculations as approximations to the true power. Most importantly, it should be noted that the substantive conclusions drawn from our data do not depend on the particular level of alpha chosen, but would also be obtained with the conventional level of $\alpha=.05$ (and $1-$ $\beta>$.999).

3. The log-likelihood goodness-of-fit statistic $G^{2}$ is asymptotically chi-square distributed with degrees of freedom indicated in parentheses (see Hu \& Batchelder, 1994, for details). If $H_{0}$ is valid, the expected value for $G^{2}$ is equal to the number of degrees of freedom. All modelbased statistical analyses reported in this article were conducted using the Apple Tree program by Rothkegel (1996).

4. Responses to Phase 2 words are included in Table 2 to make all our data available, even though they are not analyzed in typical process dissociation experiments (such as the present Experiment 1). 


\section{APPENDIX}

Table A1

Frequencies of Phase 1, Phase 2, and New Responses for the Source Monitoring Groups in Experiment 1 as a Function of Condition and Item Source

\begin{tabular}{|c|c|c|c|c|c|c|c|c|}
\hline \multirow[b]{2}{*}{ Response } & \multicolumn{4}{|c|}{ Inclusion } & \multicolumn{4}{|c|}{ Exclusion } \\
\hline & Anagram & Read & New & Phase 2 & Anagram & Read & New & Phase 2 \\
\hline Phase 1 & 287 & 156 & 53 & 59 & 275 & 146 & 72 & 69 \\
\hline Phase 2 & 43 & 57 & 22 & 271 & 55 & 79 & 33 & 264 \\
\hline New & 70 & 187 & 325 & 70 & 70 & 175 & 295 & 67 \\
\hline
\end{tabular}

Table A2

Frequencies of Beginning, Famous, and No Responses for the Source Monitoring Groups in Experiment 2 as a Function of Condition and Item Source

\begin{tabular}{lccccccc}
\hline & \multicolumn{3}{c}{ Inclusion } & & \multicolumn{3}{c}{ Exclusion } \\
\cline { 2 - 4 } \cline { 6 - 8 } Response & Beginning & No & Famous & & Beginning & No & Famous \\
\hline Beginning & 243 & 103 & 149 & & 267 & 113 & 179 \\
Famous & 234 & 189 & 935 & & 227 & 216 & 983 \\
No & 323 & 508 & 556 & & 306 & 471 & 478 \\
\hline
\end{tabular}

(Manuscript received October 14, 1998;

revision accepted for publication November I, 1999.) 